\title{
Neuroprotective effect of ginsenoside Rg1 prevents cognitive impairment induced by isoflurane anesthesia in aged rats via antioxidant, anti-inflammatory and anti-apoptotic effects mediated by the PI3K/AKT/GSK-3 $\beta$ pathway
}

\author{
YINI ZHANG ${ }^{1}$, ZHAO ZHANG $^{2}$, HAITANG WANG $^{3}$, NAN CAI $^{1}$, SHUANG ZHOU $^{1}$, YAOPING ZHAO ${ }^{1}$, \\ XUE CHEN $^{1}$, SHAOQIANG ZHENG ${ }^{1}$, QI SI ${ }^{1}$ and WEI ZHANG ${ }^{1}$ \\ ${ }^{1}$ Department of Anesthesiology, Beijing Jishuitan Hospital, Beijing 100035; \\ ${ }^{2}$ Beidaihe Sanatorium of Beijing Military Command, Qinhuangdao, Hebei 066000; ${ }^{3}$ Department of Anesthesiology, \\ Nanfang Hospital, Southern Medical University, Guangzhou, Guangdong 510515, P.R. China
}

Received June 7, 2015; Accepted May 25, 2016

DOI: $10.3892 / \mathrm{mmr} .2016 .5556$

\begin{abstract}
Ginsenoside $\operatorname{Rg} 1$ is the primary active substance in ginseng, and it has multiple pharmacological actions. Investigations on the pharmacologic action of ginsenoside Rg1 have developed, with a particular focus on the regulation of metabolism. The present study hypothesized that the neuroprotective effects of ginsenoside Rg1 prevent cognitive impairment induced by isoflurane anesthesia via antioxidant, anti-inflammatory and anti-apoptotic effects, mediated by the phosphoinositide 3-kinase (PI3K)/AKT/glycogen synthase kinase-3 $\beta$ (GSK-3 $\beta$ ) pathway in aged rats. Sprague-Dawley rats were divided into isoflurane and ginsenoside $\mathrm{Rg} 1$ groups and were treated with $20 \mathrm{mg} / \mathrm{kg}$ ginsenoside $\mathrm{Rg} 1$ for 7 days. Morris water maze was performed to analyze the cognitive function of the rats. Enzyme-linked immunosorbent assays were used to analyze the levels of malondialdehyde, glutathione, interleukin (IL)-1 $\beta$, IL-6 and caspase 3 . The protein expression levels of AKT, GSK 3 $\beta$, p21WAF1/CIP1 and p53 were measured using western blot analysis. Ginsenoside $\operatorname{Rg} 1$ significantly improved cognitive function, and exhibited antioxidant and anti-inflammatory effects, demonstrating the neuroprotective effects of ginsenoside $\mathrm{Rg} 1$ against the effect of isoflurane anesthesia in the rats. In addition, ginsenoside Rg1 significantly reduced caspase-3 activity, upregulated the expression of PI3K/AKT/GSK-3 $\beta$ and downregulated the mRNA expression levels of p21WAF1/CIP1 and p53 in the
\end{abstract}

Correspondence to: Dr Wei Zhang, Department of Anesthesiology, Beijing Jishuitan Hospital, 31 Xinjiekou East Street, Xicheng, Beijing 100035, P.R. China

E-mail: weizhang26h@163.com

Key words: ginsenoside $\operatorname{Rg} 1$, cognitive impairment, isoflurane anesthesia, phosphoinositide 3-kinase/AKT/glycogen synthase kinase $3 \beta$ aged rats exposed to isoflurane anesthesia. The data obtained in the present study provided evidence that the neuroprotective effects of ginsenoside Rg1 prevented the cognitive impairment induced by isoflurane anesthesia via antioxidant, anti-inflammatory and anti-apoptotic effects, mediated by the PI3K/AKT/GSK-3 $\beta$ pathway.

\section{Introduction}

A graying society is an indispensable and significant link during humthean indispensable (1). A graying society is a population structure in which the aging population has reached or exceeded a certain proportion (1). According to the traditional standard of the United Nations, a region where the population of individuals aged $>60$ years old is $10 \%$ of the total population can be regarded as entering into a graying society (2). However, in the current standard, it refers to a population of individuals, in which those aged $>65$ years old represent $7 \%$ of the total population (3). In 2002, China became a graying society. As age increases, the elderly are more susceptible to various types of diseases due to their unique physiological characteristics, which introduce severe challenges for medical treatment and the social insurance industry (4). It is important to seriously consider these problems, and there is interest in postoperative cognitive dysfunction (POCD), specifically in gerontal patients (5).

Isoflurane has lasting and severe effects on cognitive function. In particular, it can affect learning and memory function in sea horses $(4,6)$. Isoflurane is a novel inhalation anesthetic, which is frequently used clinically (7). It can inhibit cholinergic neurons from transmitting signals and induces anesthetic effects via the N-methyl-D aspartic acid receptor, which affect learning and memory function $(7,8)$.

Ginseng is a type of traditional Chinese medicine, which is widely used for the promotion of health and treatment of diseases (9). Ginseng, marten and cartialgenous are also collectively known as 'the three treasures' in northeast China. There are several ginsenosides, including Ro, Ra, Ral-2, Rbl-3, Rc, Rd, $\mathrm{Re}, \mathrm{Rf}$ and $\mathrm{Rg} 1$ (10). Of the ginseng glycosides, ginsenoside Rgl 
has anti-oxidative and anti-apoptotic functions and other neuroprotective effects (10). Previous experiments have indicated that ginsenoside Rg1 can improve, consolidate and recover memory in cognitive impairment caused by quinolinic acid, $\mathrm{Pb}$, dexamethasone, anisodine, ethylene and ethyl alcohol $(11,12)$. In addition, it can improve impairment of learning and memory function caused by scopolamine and morphine (13). The aims of the present study were to detect whether the neuroprotective effect of ginsenoside Rg1 prevents cognitive impairment induced by isoflurane anesthesia via antioxidant, anti-inflammatory and anti-apoptotic effects, and whether these are mediated by the phosphoinositide 3-kinase (PI3K)/AKT/glycogen synthase kinase-3 $\beta$ (GSK-3 $\beta$ ) pathway in aged rats.

\section{Materials and methods}

Reagents. Ginsenoside Rg1 ( $\geq 90 \%$ in high performance liquid chromatography) was purchased from Sigma-Aldrich (St. Louis, MO, USA; its chemical structure is shown in Fig. 1. Isoflurane, the interleukin (IL)-1 $\beta$ kit (cat. no. H002), IL-6 kit (cat. no. H007), malondialdehyde (MDA) kit (cat. no. A003-1), caspase-3 kit (cat. no. H076) and glutathione (GSH) kit (cat. no. A006-2) were purchased from Nanjing Jiancheng Bioengineering Institute (Nanjing, China). The bicinchoninic acid assay (BCA) assay was purchased from Wuhan Boster Bio-engineering Co., Ltd. (Wuhan, China).

Animals and ethical statement. Male, 3-month-old Sprague-Dawley (SD) rats were purchased from the Medical and Laboratory Animal Center of Beijing Jishuitan Hospital (Beijing, China) and housed at a temperature $\left(23 \pm 1^{\circ} \mathrm{C}\right)$ in a light-controlled room (12-h light/dark cycle), with a relative humidity of 50-70\% and free access to water and food. All SD rats were treated, according to the guidelines of the Guide for the Care and Use of Laboratory Animals of Beijing Jishuitan Hospital and the study was approved by the ethics committee of Beijing Jishuitan Hospital.

Exposure to anesthesia and grouping. The rats were randomly divided into three groups: Control, isoflurane and ginsenoside $\operatorname{Rg} 1$ ( $n=10$ in each group). The SD rats in the ginsenoside $\mathrm{Rg} 1$ group were treated with $20 \mathrm{mg} / \mathrm{kg}$ ginsenoside $\mathrm{Rg} 1$ for 7 days (14) by intraperitoneal injection. After $12 \mathrm{~h}$, the rats in the isoflurane and ginsenoside $\mathrm{Rg} 1$ groups were exposed to $1.3 \%$ isoflurane (Nanjing Jiancheng Bioengineering Institute) in a humidified $30 \%$ oxygen carrier gas for $4 \mathrm{~h}$. The SD rats in the control group were exposed to humidified $30 \%$ oxygen without isoflurane for $4 \mathrm{~h}$.

Morris water maze performance. A round pool, with a diameter of $150 \mathrm{~cm}$ and depth of $50 \mathrm{~cm}$, was filled with $24^{\circ} \mathrm{C}$ opaque water to the top of a movable, clear $15-\mathrm{cm}$-diameter platform (1.5 cm above water). Motion detection software (Actimetrics software, Evanston, IL, USA; version ACT-556) was used to record the swimming motions and analyze the results. Each rat was wiped dry and kept warm, and returned to their cage with free access to food. Following isoflurane exposure, trials were performed for 4 days, and the spatial information of every rat was analyzed. The pool had a dark black surround, which was used to prevent confounding visual cues. All rats were placed

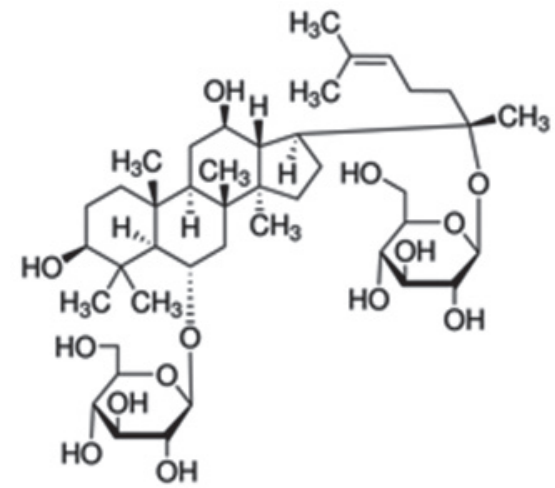

Figure 1. Chemical structure of ginsenoside Rg1.

in a fixed position in the swimming pool, facing the wall, on the 4 days. The rats were allowed to find the platform (in the third quadrant) for $20 \mathrm{sec}$, prior to being removed from the pool. The swim speed and the time of escape latency were recorded. Less time taken for a rat to reach the platform indicated a higher learning ability.

Detection of levels of MDA, GSH, IL-1 $\beta, I L-6$ and caspase- 3 using ELISA. Following isoflurane exposure, the rats in each group were sacrificed and the hippocampi were dissected for measurement of the protein levels of IL-1 $\beta$, IL-6, MDA, GSH and caspase-3 using ELISA kits (Nanjing Jiancheng Bioengineering Institute).

Western blot analysis of the protein expression of $A K T$, $G S K-3 \beta, p 21 W A F 1 / C I P 1$ and $p 53$. Hippocampal tissues were collected and were homogenized with phenylmethanesulfonyl fluoride containing radioimmunoprecipitation assay buffer and protease inhibitor cocktail (EDTA-free) on ice. The supernatant were collected and centrifuged at 13,000 $\mathrm{x} \mathrm{g}$ at $4^{\circ} \mathrm{C}$ for $30 \mathrm{~min}$, following which the BCA method (Wuhan Boster Bio-engineering, Wuhan, China) was used to analyze protein concentrations. The protein $(50 \mathrm{mg})$ was separated by SDS-PAGE and transferred onto polyvinylidene fluoride membranes (Wuhan Boster Biological Technology, Wuhan, China). The membranes were blocked using 5\% skim milk powder and incubated overnight at $4^{\circ} \mathrm{C}$ with anti-AKT ( 1:500; cat. no. sc-5298; Santa Cruz Biotechnology, Inc., Dallax, TX, USA), anti-phosphorylated (p)-AKT (1:500; cat. no. sc-293125; Santa Cruz Biotechnology, Inc.), anti-GSK-3 $\beta$ (1:2,000; cat. no. 9337; Cell Signaling Technology, Inc., Danvers, MA, USA), anti-p21WAF1/CIP1 (1:2,000; cat. no. 2947; Cell Signaling Technology, Inc.), anti-p53 (1:2,000; cat. no. 2524; Cell Signaling Technology, Inc.) and anti- $\beta$-actin antibodies (1:500; cat. no. BM0626; Wuhan Boster Biological Technology). The membranes were then incubated with anti-mouse secondary antibody (1:5,000; cat. no. BM2002; Wuhan Boster Biological Technology) at $37^{\circ} \mathrm{C}$ for $1 \mathrm{~h}$ and visualized using an enhanced chemiluminescence detection system (Thermo Fisher Scientific, Inc., Waltham, MA, USA) and expression was quantified using a gel inage analysis system (Thermo Fisher Scientific, Inc.).

Statistical analysis. All data are presented as the mean \pm standard error of the mean, and analysis was performed using 
A

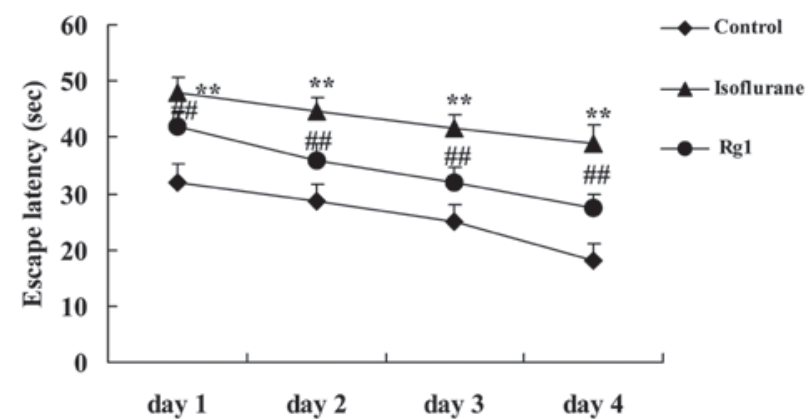

C

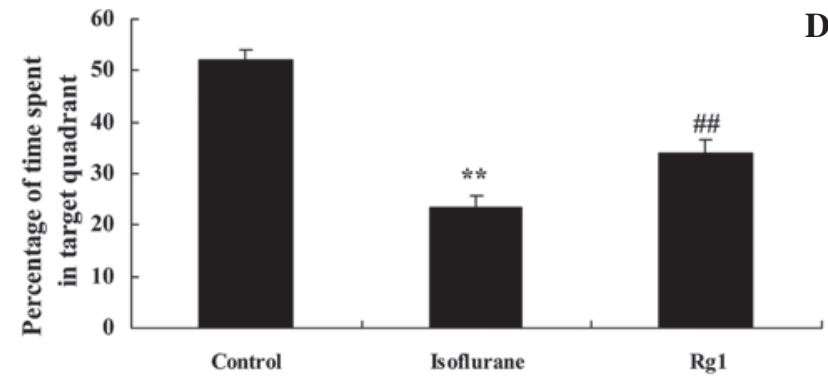

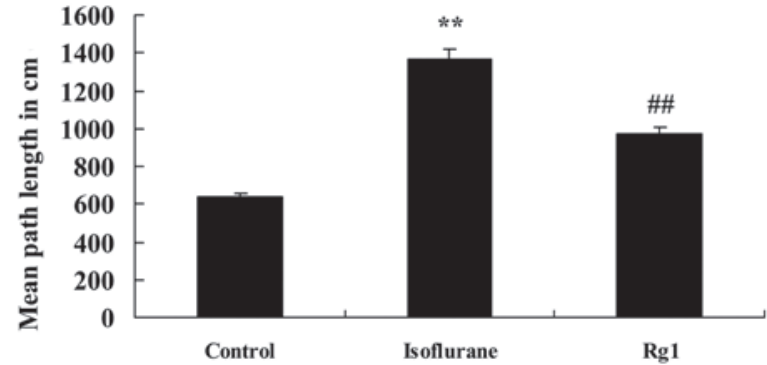

D

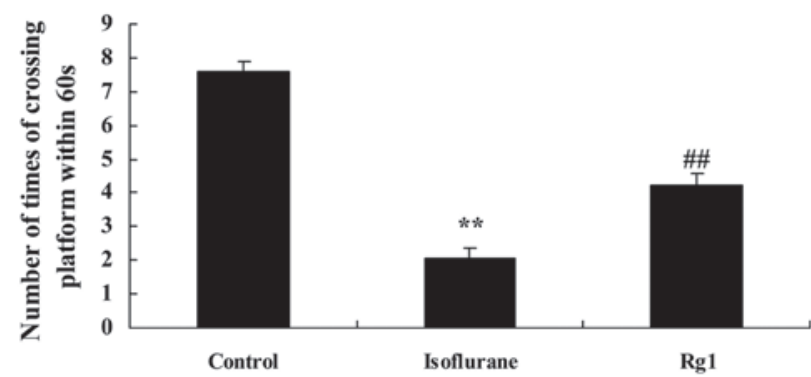

Figure 2. Neuroprotective effect of ginsenoside $\mathrm{Rg} 1$ on cognitive function in aging rats exposed to isoflurane anesthesia. Neuroprotective effects of ginsenoside Rg1 on (A) escape latency, (B) path length, (C) mean swim speed and (D) duration of swimming were examined in aging rats exposed to isoflurane anesthesia. Data are presented as the mean \pm standard error of the mean. ${ }^{* *} \mathrm{P}<0.01$, compared with the control group; ${ }^{\# \#} \mathrm{P}<0.01$, compared with the isoflurane group.

A

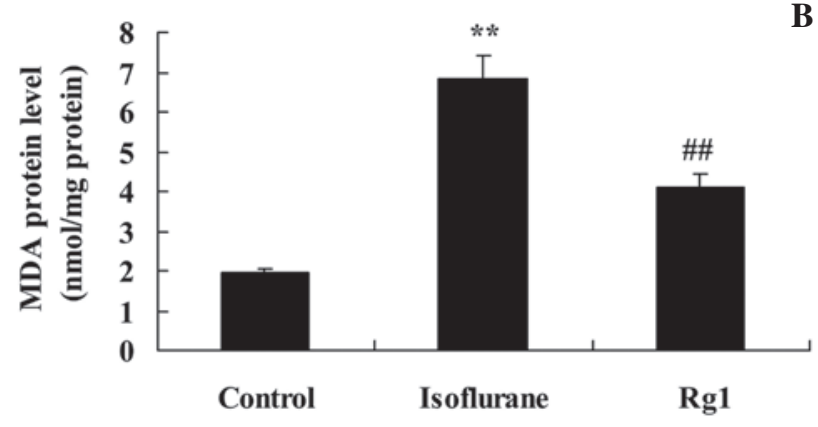

B

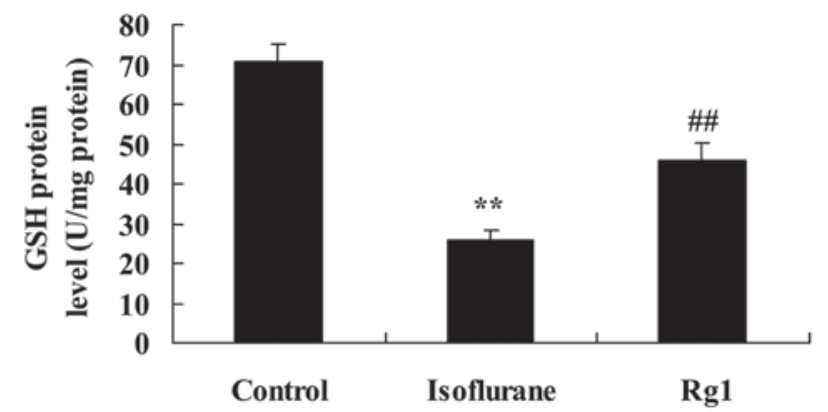

Figure 3. Antioxidant effect of ginsenoside Rg1 in aging rats exposed to isoflurane anesthesia. Effects of ginsenoside Rg1 on the protein levels of (A) MDA and (B) GSH were examined in aging rats exposed to isoflurane anesthesia. Data are presented as the mean \pm standard error of the mean. ${ }^{* *} \mathrm{P}<0.01$, compared with the control group; ${ }^{\# \#} \mathrm{P}<0.01$, compared with the isoflurane group. MDA, malondialdehyde; GSH, glutathione.

SPSS 17.0 (SPSS, Inc., Chicago, IL, USA). One-way analysis of variance was used for comparison of mean values across the groups. $\mathrm{P}<0.05$ was considered to indicate a statistically significant difference.

\section{Results}

Neuroprotective effects of ginsenoside Rgl on cognitive function in isoflurane anesthesia-exposed aged rats. As shown in Fig. 2A and B, the escape latency and path length of the control group were lower, compared with those of the isoflurane anesthesia group following the Morris water sessions. Ginsenoside Rg1 effectively inhibited the increased escape latency and path length in the aged rats exposed to isoflurane anesthesia (Fig. 2A and B). The mean swim speed and swimming durations were significantly inhibited in the aged rats exposed to isoflurane anesthesia, compared with the control group (Fig. 2C and D). However, ginsenoside Rg1 effectively elevated the suppression of mean swim speed and extended the duration of swimming in the rats exposed to isoflurane anesthesia (Fig. 2C and D).

Antioxidant effects of ginsenoside Rgl in aging rats exposed to isoflurane anesthesia. As shown in Fig. 3A and B, the rats in the isoflurane groups had significantly increased protein levels of MDA and decreased protein levels of GSH, compared with the control group. Treatment with ginsenoside Rg1 markedly reduced the elevated MDA protein level and inhibited GSH protein level in the rats exposed to isoflurane anesthesia (Fig. 3A and B).

Anti-inflammatory effect of ginsenoside Rgl in aging rats exposed to isoflurane anesthesia. The results of the present study revealed increases in the protein levels of IL-1 $\beta$ and IL-6 
A

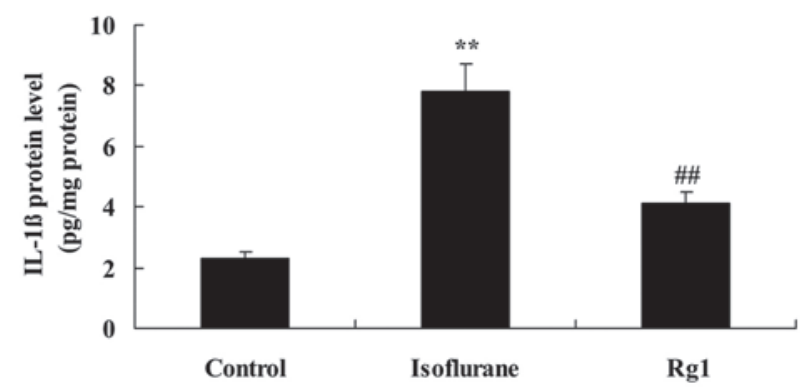

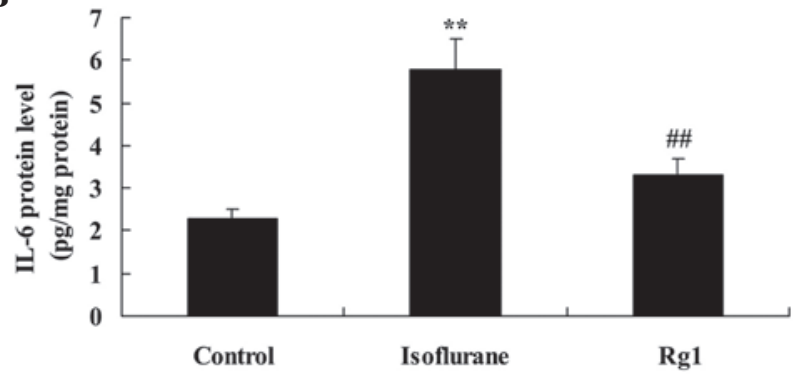

Figure 4. Anti-inflammatory effect of ginsenoside $\operatorname{Rg} 1$ in aging rats exposed to isoflurane anesthesia. Effects of ginsenoside Rg1 on the protein expression of (A) IL-1 $\beta$ and (B) IL-6 were examined in aging rats exposed to isoflurane anesthesia. Data are presented as the mean \pm standard error of the mean. ${ }^{* *} \mathrm{P}<0.01$, compared with the control group; ${ }^{\# \#} \mathrm{P}<0.01$, compared with the isoflurane group. IL, interleukin.

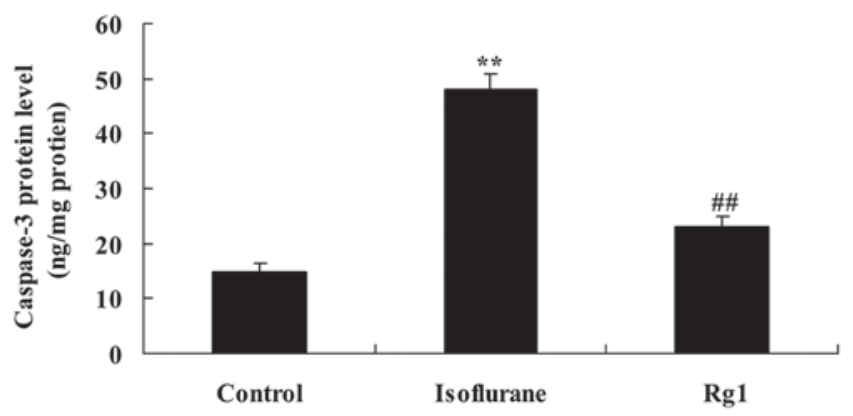

Figure 5. Anti-apoptotic effects of ginsenoside $\mathrm{Rg} 1$ in aging rats exposed to isoflurane anesthesia. The protein levels of caspase-3 were determined. Data are presented as the mean \pm standard error of the mean. ${ }^{* *} \mathrm{P}<0.01$, compared with the control group; ${ }^{\# \#} \mathrm{P}<0.01$, compared with isoflurane group.

in the isoflurane anesthesia group, compared with the control group (Fig. 4A and B). However, the isoflurane-induced protein levels of IL-1 $\beta$ and IL- 6 were significantly weakened by pretreatment with ginsenoside Rg1 (Fig. 4A and B).

Anti-apoptotic effect of ginsenoside $\mathrm{Rg} 1$ in aging rats exposed to isoflurane anesthesia. Isoflurane exposure significantly increased the protein level of caspase-3 in the aging rat, compared with the rats in the control group (Fig. 5). This increase was attenuated by ginsenoside Rg1 (Fig. 5).

Neuroprotective effect of ginsenoside $\mathrm{Rg} 1$ on $p$-AKT/AKT in isoflurane anesthesia-exposed aging rats. Compared with the rats in the control group, there was a significant decrease in the level of p-AKT/AKT of the aging rat following isoflurane exposure (Fig. 6). However, treatment with ginsenoside Rg1 effectively increased the levels of p-AKT/AKT in the aging rats exposed to isoflurane (Fig. 6).

Neuroprotective effect of ginsenoside Rgl on GSK-3 $\beta$ in aging rats exposed to isoflurane anesthesia. Compared with the rats in the control group, the protein expression of GSK-3 $\beta$ was reduced in the aging rats following exposure to isoflurane (Fig. 7). This inhibited protein expression of GSK-3 $\beta$ was elevated by treatment with ginsenoside Rg1 (Fig. 7).

Neuroprotective effect of ginsenoside Rg1 on p21WAF1/CIPI in isoflurane anesthesia-exposed aging rats. As shown in Fig. 8, the protein expression of p21WAF1/CIP1 in the isoflurane anesthesia group was higher, compared with that of the control group. Ginsenoside Rg1 treatment significantly inhibited the promotion in the protein expression of p21WAF1/CIP1 in the aging rat exposed to isoflurane anesthesia (Fig. 8).

Neuroprotective effect of ginsenoside $\mathrm{Rg} 1$ on p53 in isoflurane anesthesia-exposed aging rats. Compared with the control group, the protein expression of p53 was activated by isoflurane (Fig. 9). However, the isoflurane-induced protein expression of p53 was suppressed by pretreatment with ginsenoside $\mathrm{Rg} 1$ in the aging rats exposed to isoflurane anesthesia (Fig. 9).

\section{Discussion}

POCD is an acute reversible abalienation syndrome that occurs following surgery with anesthesia (15). It often occurs in the elderly several days or several weeks following surgery, with a decline in memory, comprehension and concentration, and a decrease in social adaptation ability, possibly leading to the loss of independent living and a permanent cognitive disorder (16). POCD was originally identified in gerontal patients following cardiac surgery (17), however, it has been found in patients who have not had cardiac surgery with higher rates of morbidity. There are several risk factors for POCD during the early post-operative period, including history of surgery with anesthesia, low levels of education, secondary surgery, postoperative infection and respiratory system complications, whereas advanced age is the predominant risk factor during the later post-operative period (18). Compared with younger adults, the brain cells of the elderly are atrophied, with seriously decreased cranial capacity, enlarged ventricles, and alterations in cranial nerve distribution and neurotransmitter types (19). As a result, gerontal patients are more sensitive to nerve cell damage caused by surgery and anesthesia (20). Furthermore, postoperative pain can affect cognitive function during early period, however, chronic pain prior to surgery has no effect on the results of cognitive function assessments (21). The results of the present study suggested that the neuroprotective effects of ginsenoside Rg1 improved cognitive function in the aging rats exposed to isoflurane anesthesia. 
A

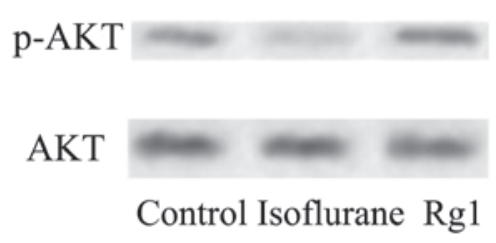

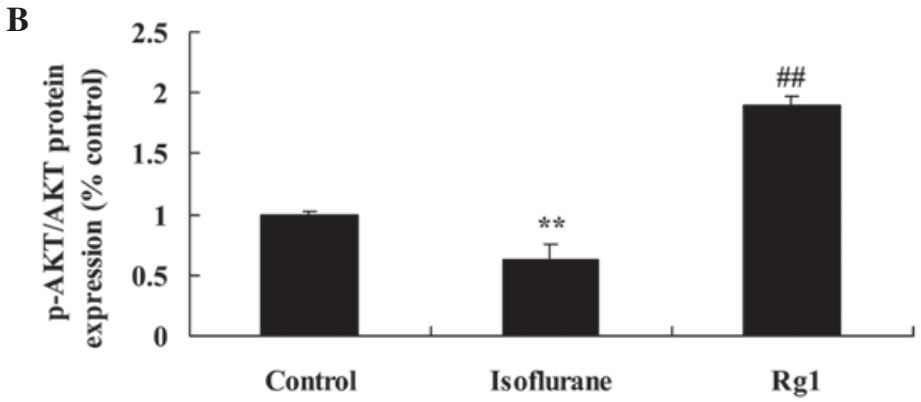

Figure 6. Neuroprotective effect of ginsenoside $\operatorname{Rg} 1$ on p-AKT/AKT in isoflurane anesthesia exposed aging rats. The effects of ginsenoside Rg1 on the protein expression levels of p-AKT and AKT were determined using (A) western blot analysis, and (B) statistical analysis of the protein expression levels of p-AKT and AKT was performed. Data are presented as the mean \pm standard error of the mean. ${ }^{* *} \mathrm{P}<0.01$, compared with the control group; ${ }^{\# \#} \mathrm{P}<0.01$, compared with the isoflurane group.

A

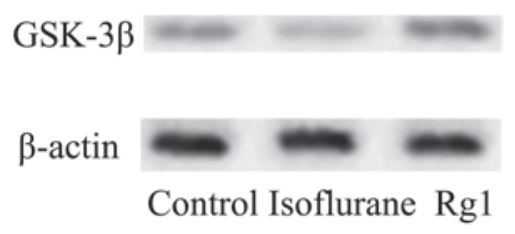

B

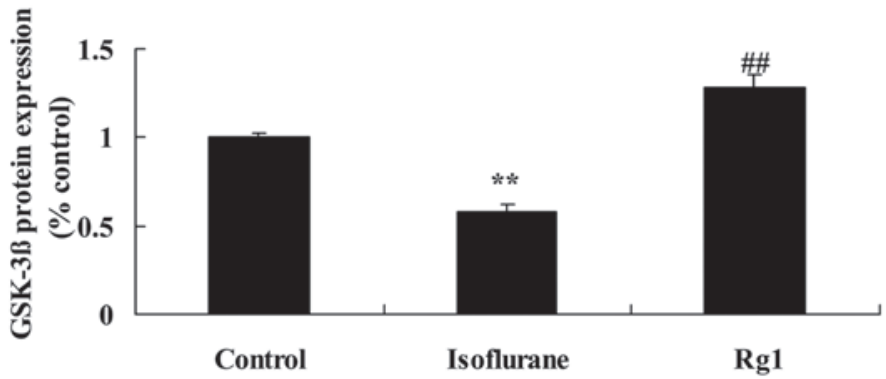

Figure 7. Neuroprotective effect of ginsenoside Rg1 on GSK-3 $\beta$ in aging rats exposed to isoflurane anesthesia. (A) Effect of ginsenoside Rg1 on the protein expression of GSK-3 $\beta$, determined using western blot analysis. (B) Statistical analysis of the protein expression levels. Data are presented as the mean \pm stan-

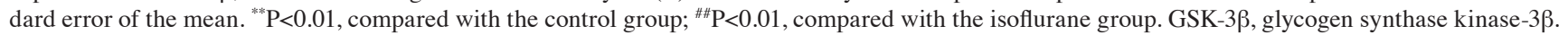

A

\section{p21WAF1/CIP1}

$\beta$-actin

Control Isoflurane $\mathrm{Rg} 1$
B

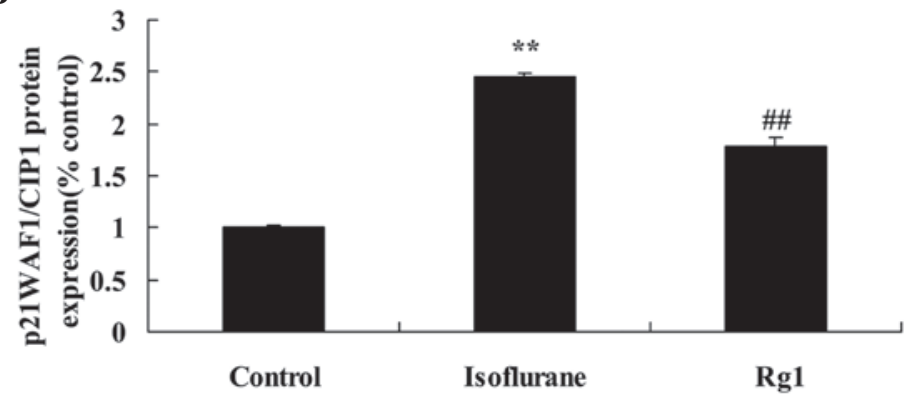

Figure 8. Neuroprotective effect of ginsenoside Rg1 on p21WAF1/CIP1 in aging rats exposed to isoflurane anesthesia. (A) Neuroprotective effect of ginsenoside $\mathrm{Rg} 1$ on the protein expression of p21WAF1/CIP1, determined using western blot analysis. (B) Statistical analysis of the protein expression of p21WAF1/CIP1. Data are presented as the mean \pm standard error of the mean. ${ }^{* *} \mathrm{P}<0.01$, compared with the control group; ${ }^{* \prime \prime} \mathrm{P}<0.01$, compared with the isoflurane group.

A

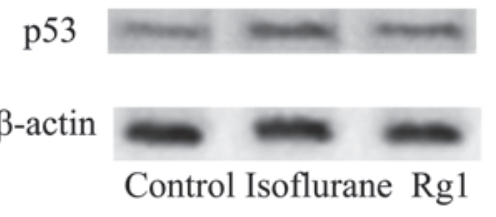

B

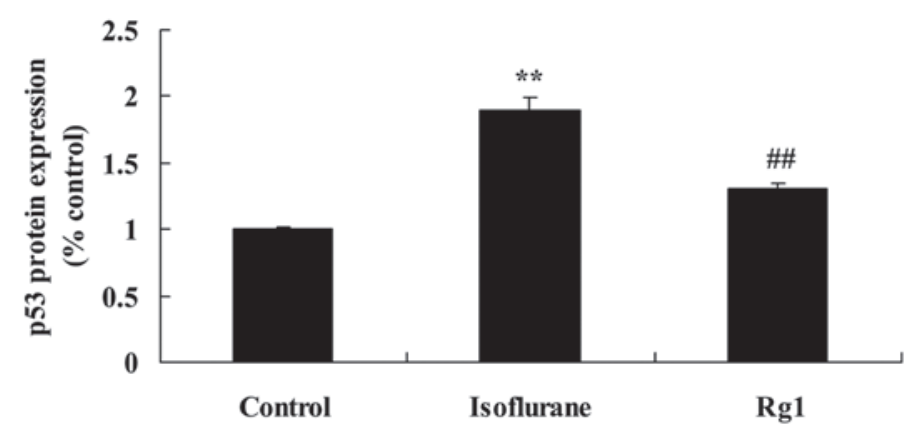

Figure 9. Neuroprotective effect of ginsenoside $\mathrm{Rg} 1$ on p53 in aging rats exposed to isoflurane anesthesia. (A) Neuroprotective effect of ginsenoside Rg1 on the protein expression of p53, determined using western blot analysis. (B) Statistical analysis of the protein expression of p53. Data are presented as the mean \pm standard error of the mean. ${ }^{* *} \mathrm{P}<0.01$, compared with the control group; ${ }^{\# \#} \mathrm{P}<0.01$, compared with the isoflurane group. 
In previous years, a number of studies have indicated that the inflammatory response inside the brain and oxidative stress are of significant importance in the pathogenesis of cognitive impairment (22). Several reports have confirmed that inflammation contributes to pathological injury in various ways. For example, inflammation damages the integrity of vascular function and contributes to the pathogenesis of cognitive impairment diseases, including POCD (23). Treatment of this disease through targeting this pathomechanism can significantly improve neuronal injury and modify nerve fiberdamage to relieve the inflammatory response within the brain, improving learning and memory abilities $(23,24)$. Therefore, there has been substantial investigation on the association between oxidative stress and cognitive impairment (25). Free radicals have high levels of activity and potent oxidative effects, which can injure biomacromolecules and multiple components of cells. In addition, they are harmful to nerve cells, causing alterations, including lipofuscin deposition, age pigment increase and vacuolar degeneration within the cells (23). The present study found that ginsenoside Rg1 exhibited antioxidant and anti-inflammatory effects in the treatment of isoflurane-induced cognitive impairment in aging rats. Yang et al also reported that ginsenoside Rg1 exerts anti-inflammatory and anti-apoptotic properties in rats with ischemia-reperfusion injury (26), and Yu et al (9) suggested that ginsenoside Rg1 ameliorates oxidative stress in streptozotocin-induced diabetic rats.

Caspase-3 is the most important protease during apoptosis and is widely used for detecting cell apoptosis (27). Used as an apoptotic detection indicator, caspase-3 has confirmed that sevoflurane and isoflurane exposure in juvenile rats may lead to increased brain cell apoptosis, and that prolonging the duration of anesthesia increases the number of apoptotic cells (28). These findings indicate that there is causal association between the severity of brain cell apoptosis and the duration of drug treatment under these experimental conditions. Of note, ginsenoside Rg1 exerted anti-apoptotic effects and inhibited the expression of caspase- 3 in the aging rats exposed to isoflurane anesthesia in the present study. Yang et al also showed that ginsenoside Rg1 suppresses the inflammation and level of caspase- 3 in neuron cells in rats with cerebral ischemia-reperfusion injury (26).

The PI3K/AKT signal transduction pathway is important for membrane receptor signal transduction into cells, and it reduces cell apoptosis due to ischemia-reperfusion injury in organs, including the heart, kidney and liver (29). GSK-3 is a multifunctional serine/threonine protein kinase involved in cell differentiation, proliferation and apoptosis, with the exception of glycometabolism (30). The PI3K/AKT pathway is involved in regulating the gene expression of myeloid cell leukemia-1 (Mcl-1) in multiple types of tumor and cell line (30). AKT can phosphorylate $\mathrm{B}$ cell lymphoma $(\mathrm{Bcl})$-associated $\mathrm{X}$ protein and forms a heterodimer with Bcl-extra large and Mcl-1 in the cytoplasm, leading to decreased mitochondrial membrane translocation and cell apoptosis. GSK-3 $\beta$ can phosphorylate Mcl-1 to increase the ubiquitination of Mcl-1 and decrease the induction of apoptosis. In addition, AKT affects the stability of Mcl-1 by negatively regulating GSK-3 $\beta$ to inhibit apoptosis (31). In the present study, ginsenoside Rg1 activated the PI3K/AKT/GSK-3 $\beta$ pathway in aging rats exposed to isoflurane anesthesia. Wang et al also found that ginsenoside $\mathrm{Rg} 1$ regulates innate immune responses via the PI3K/AKT pathway (32), and Song et al reported that ginsenoside Rg1 attenuates spatial memory impairment through the GSK3 $\beta$ signaling pathway in rats (33).

The p53 gene is a reactive gene located upstream and affected by cell DNA damage. If cells are exposed to external injury, the p53 gene is activated, and the protein expression of p53 mediates cell cycle arrest by activating a series of downstream cells, or by immediately combining with single or double stranded DNA to induce self-repair of DNA in cells (34). As the direct downstream mediator of p53, p21WAF1/CIP1 is important in adjusting the cell cycle by combining and restraining cyclin dependent kinase (35). In addition to cell cycle arrest, p21WAF1/CIP1 also inhibits p53 mediation and the apoptosis mediated by p53, and under certain specific conditions, it also promote cell apoptosis (36). On analyzing the expression and behavior changes of nerve cell apoptosis and p21WAF1/CIP1, p53 protein, the results indicate that it presents negative correlation about the expression and behavior changes of the former two, otherwise, cell apoptosis and p21WAF1/CIP1, p53 protein express more, the injury of cognitive function is more serious (37). In the present study, treatment with ginsenoside Rg1 suppressed the protein expression of $\mathrm{p} 21 \mathrm{WAF} 1 / \mathrm{CIP} 1$ in aging rats exposed to isoflurane anesthesia. Zhu et al also reported that ginsenoside Rg1 prevented cognitive impairment through the inhibition of p21WAF1/CIP1 in D-galactose-induced aging rat (11).

In conclusion, the present study demonstrated the neuroprotective effects of ginsenoside Rg1 in preventing the cognitive impairment induced by isoflurane anesthesia via antioxidant, anti-inflammatory and anti-apoptotic effects mediated by the $\mathrm{PI} 3 \mathrm{~K} / \mathrm{AKT} / \mathrm{GSK}-3 \beta$ pathway in aging rats.

\section{References}

1. Lin D and Zuo Z: Isoflurane induces hippocampal cell injury and cognitive impairments in adult rats. Neuropharmacology 61: 1354-1359, 2011.

2. Spitzer WJ and Davidson KW: Future trends in health and health care: Implications for social work practice in an aging society. Soc Work Health Care 52: 959-986, 2013.

3. Tachibana S, Hayase T, Osuda M, Kazuma S and Yamakage M: Recovery of postoperative cognitive function in elderly patients after a long duration of desflurane anesthesia: A pilot study. J Anesth 29: 627-630, 2015.

4. Wang W, Wang Y, Wu H, Lei L, Xu S, Shen X, Guo X, Shen R, Xia X, Liu Y and Wang F: Postoperative cognitive dysfunction: Current developments in mechanism and prevention. Med Sci Monit 20: 1908-1912, 2014.

5. Liu J, Wang P, Zhang X, Zhang W and Gu G: Effects of different concentration and duration time of isoflurane on acute and long-term neurocognitive function of young adult C57BL/6 mouse. Int J Clin Exp Pathol 7: 5828-5836, 2014.

6. Velly L, Mantz J and Bruder N: Dual effects of isoflurane on neuronal proliferation/differentiation: A substrate to impaired cognitive function? Anesthesiology 118: 487-489, 2013.

7. Stratmann G, Sall JW, May LD, Loepke AW and Lee MT: Beyond anesthetic properties: The effects of isoflurane on brain cell death, neurogenesis and long-term neurocognitive function. Anesth Analg 110: 431-437, 2010.

8. Culley DJ, Xie Z and Crosby G: General anesthetic-induced neurotoxicity: An emerging problem for the young and old? Curr Opin Anaesthesiol 20: 408-413, 2007.

9. Yu HT, Zhen J, Pang B, Gu JN and Wu SS: Ginsenoside Rg1 ameliorates oxidative stress and myocardial apoptosis in streptozotocin-induced diabetic rats. J Zhejiang Univ Sci B 16: 344-354, 2015 
10. Lu D, Zhu LH, Shu XM, Zhang CJ, Zhao JY, Qi RB, Wang HD and Lu DX: Ginsenoside Rg1 relieves tert-Butyl hydroperoxide-induced cell impairment in mouse microglial BV2 cells. J Asian Nat Prod Res 17: 930-945, 2015.

11. Zhu J, Mu X, Zeng J, Xu C, Liu J, Zhang M, Li C, Chen J, Li T and Wang Y: Ginsenoside Rg1 prevents cognitive impairment and hippocampus senescence in a rat model of D-galactose-induced aging. PLoS One 9: e101291, 2014.

12. Fang F, Chen X, Huang T, Lue LF, Luddy JS and Yan SS: Multi-faced neuroprotective effects of Ginsenoside Rg1 in an Alzheimer mouse model. Biochim Biophys Acta 1822: 286-292, 2012.

13. Wang $\mathrm{Y}, \mathrm{Kan} \mathrm{H}$, Yin $\mathrm{Y}, \mathrm{Wu} \mathrm{W}, \mathrm{Hu} \mathrm{W}$, Wang $\mathrm{M}, \mathrm{Li} \mathrm{W}$ and Li W: Protective effects of ginsenoside Rg1 on chronic restraint stress induced learning and memory impairments in male mice. Pharmacol Biochem Behav 120: 73-81, 2014.

14. Lee B, Shim I, Lee H and Hahm DH: Effect of ginsenoside Re on depression- and anxiety-like behaviors and cognition memory deficit induced by repeated immobilization in rats. J Microbiol Biotechnol 22: 708-720, 2012.

15. Rossi A, Burkhart C, Dell-Kuster S, Pollock BG, Strebel SP, Monsch AU, Kern C and Steiner LA: Serum anticholinergic activity and postoperative cognitive dysfunction in elderly patients. Anesth Analg 119: 947-955, 2014.

16. van Harten AE, Scheeren TW and Absalom AR: A review of postoperative cognitive dysfunction and neuroinflammation associated with cardiac surgery and anaesthesia. Anaesthesia 67: 280-293, 2012.

17. Riedel B, Browne K and Silbert B: Cerebral protection: Inflammation, endothelial dysfunction and postoperative cognitive dysfunction. Curr Opin Anaesthesiol 27: 89-97, 2014.

18. Zheng XU, Ma Z and Gu X: Plasma levels of tumor necrosis factor- $\alpha$ in adolescent idiopathic scoliosis patients serve as a predictor for the incidence of early postoperative cognitive dysfunction following orthopedic surgery. Exp Ther Med 9: 1443-1447, 2015.

19. Marshall JW and Ridley RM: Assessment of cognitive and motor deficits in a marmoset model of stroke. ILAR J 44: 153-160, 2003

20. Li Y, Wang S, Ran K, Hu Z, Liu Z and Duan K: Differential hippocampal protein expression between normal aged rats and aged rats with postoperative cognitive dysfunction: A proteomic analysis. Mol Med Rep 12: 2953-2960, 2015.

21. Jo YY, Kim JY, Lee MG, Lee SG and Kwak HJ: Changes in cerebral oxygen saturation and early postoperative cognitive function after laparoscopic gastrectomy: A comparison with conventional open surgery. Korean J Anesthesiol 69: 44-50, 2016.

22. Liu C and Han JG: Advances in the mechanisms and early warning indicators of the postoperative cognitive dysfunction after the extracorporeal circulation. Zhongguo Yi Xue Ke Xue Yuan Xue Bao 37: 101-107, 2015.

23. Ogasawara K, Yamadate K, Kobayashi M, Endo H, Fukuda T, Yoshida K, Terasaki K, Inoue T and Ogawa A: Effects of the free radical scavenger, edaravone, on the development of postoperative cognitive impairment in patients undergoing carotid endarterectomy. Surg Neurol 64: 309-313; discussion 313-314, 2005.

24. Tan H, Cao J, Zhang J and Zuo Z: Critical role of inflammatory cytokines in impairing biochemical processes for learning and memory after surgery in rats. J Neuroinflammation 11: 93, 2014.
25. An LN, Yue Y, Guo WZ, Miao YL, Mi WD, Zhang H, Lei ZL, Han SJ and Dong L: Surgical trauma induces iron accumulation and oxidative stress in a rodent model of postoperative cognitive dysfunction. Biol Trace Elem Res 151: 277-283, 2013.

26. Yang Y, Li X, Zhang L, Liu L, Jing G and Cai H: Ginsenoside Rg1 suppressed inflammation and neuron apoptosis by activating PPAR $\gamma / \mathrm{HO}-1$ in hippocampus in rat model of cerebral ischemia-reperfusion injury. Int J Clin Exp Pathol 8: 2484-2494, 2015.

27. Miyamoto A, Miyauchi H, Kogure T, Miyawaki A, Michikawa T and Mikoshiba K: Apoptosis induction-related cytosolic calcium responses revealed by the dual FRET imaging of calcium signals and caspase- 3 activation in a single cell. Biochem Biophys Res Commun 460: 82-87, 2015.

28. Li Y, Liu C, Zhao Y, Hu K, Zhang J, Zeng M, Luo T, Jiang W and Wang H: Sevoflurane induces short-term changes in proteins in the cerebral cortices of developing rats. Acta Anaesthesiol Scand 57: 380-390, 2013.

29. Chen L, Xiang Y, Kong L, Zhang X, Sun B, Wei X and Liu H: Hydroxysafflor yellow A protects against cerebral ischemia-reperfusion injury by anti-apoptotic effect through PI3K/Akt/GSK3 $\beta$ pathway in rat. Neurochem Res 38: 2268-2275, 2013.

30. Son YO, Pratheeshkumar P, Wang L, Wang X, Fan J, Kim DH, Lee JY, Zhang Z, Lee JC and Shi X: Reactive oxygen species mediate $\mathrm{Cr}(\mathrm{VI})$-induced carcinogenesis through PI3K/AKT-dependent activation of GSK-3 $/ / \beta$-catenin signaling. Toxicol Appl Pharmacol 271: 239-248, 2013.

31. Yamaguchi K, Lee SH, Eling TE and Baek SJ: Identification of nonsteroidal anti-inflammatory drug-activated gene (NAG-1) as a novel downstream target of phosphatidylinositol 3-kinase/AKT/GSK-3beta pathway. J Biol Chem 279: 49617-49623, 2004.

32. Wang Y, Liu Y, Zhang XY, Xu LH, Ouyang DY, Liu KP, Pan H, $\mathrm{He} \mathrm{J}$ and $\mathrm{He} \mathrm{XH}$ : Ginsenoside $\mathrm{Rg} 1$ regulates innate immune responses in macrophages through differentially modulating the NF-kappaB and PI3K/Akt/mTOR pathways. Int Immunopharmacol 23: 77-84, 2014

33. Song XY, Hu JF, Chu SF, Zhang Z, Xu S, Yuan YH, Han N, Liu Y, Niu F, He X and Chen NH: Ginsenoside Rg1 attenuates okadaic acid induced spatial memory impairment by the GSK3//tau signaling pathway and the $A \beta$ formation prevention in rats. Eur J Pharmacol 710: 29-38, 2013.

34. Hill R, Madureira PA, Waisman DM and Lee PW: DNA-PKCS binding to $\mathrm{p} 53$ on the p21WAF1/CIP1 promoter blocks transcription resulting in cell death. Oncotarget 2: 1094-1108, 2011.

35. Aliouat-Denis CM, Dendouga N, Van den Wyngaert I, Goehlmann H, Steller U, van de Weyer I, Van Slycken N, Andries L, Kass S, Luyten W, et al: p53-independent regulation of p21Waf1/Cip1 expression and senescence by Chk2. Mol Cancer Res 3: 627-634, 2005.

36. Huang L, Sowa Y, Sakai T and Pardee AB: Activation of the p21WAF1/CIP1 promoter independent of p53 by the histone deacetylase inhibitor suberoylanilide hydroxamic acid (SAHA) through the Sp1 sites. Oncogene 19: 5712-5719, 2000.

37. Alpan RS and Pardee AB: p21WAF1/CIP1/SDI is elevated through a p53-independent pathway by mimosine. Cell Growth Differ 7: 893-901, 1996. 